\title{
Jornadas de Otoño 2009: Avances en Tromboembolismo Pulmonar
}

\author{
Fall Meeting 2009: Advances in Pulmonary Tromboembolism
}

El diagnóstico de tromboembolismo pulmonar (TEP) siempre involucra un desafio para el médico. En muchas ocasiones, la sobrevida del paciente depende de su rápido reconocimiento y del pronto inicio de la terapia.

Durante las $28^{a}$ Jornadas de Otoño se discutió el diagnóstico de esta entidad. Se hizo especial énfasis en la aplicación de criterios diagnósticos como la escala de Wells, que es el más usado dado su simplicidad de aplicación. Sin embargo, debe ser siempre el criterio clínico el que predomine en la toma de las decisiones con cada paciente en particular. Lo que sí está claro, es que no se debe esperar el resultado de un examen diagnóstico para iniciar terapia si se sospecha esta patología. La radiografía de tórax no sirve como elemento diagnóstico, ya que habitualmente es normal. El cintigrama de ventilación perfusión $\left(V^{\prime} / Q^{\prime}\right)$, algo olvidado, mantiene su relevancia siempre que sea normal o de alta probabilidad para TEP. La Angio TAC pulmonar permite confirmar el diagnóstico clínico demostrando elementos diagnósticos directos (defecto de llene de arteria pulmonar o de sus ramas) o indirectos (patrón en mosaico o imagen de infarto pulmonar), o permite darnos una alternativa diagnóstica como neumotórax, neumonía u otro. La resonancia magnética pulmonar no ha demostrado ser más útil en el diagnóstico de TEP que la Angio TAC. La angiografia sigue siendo el "gold standard" de esta patología. No debemos olvidar la importancia que tiene el estudio de las extremidades inferiores, buscando trombosis venosa profunda, ya sea con Ecodoppler, Venografía o TAC, tal como lo demostró el estudio PIOPED II'.

En la evaluación de los pacientes con TEP siempre se debe evaluar si tiene características de masivo, lo cual tiene implicancias en el pronóstico y manejo de los enfermos. Esta apreciación puede ser clínica, por antecedentes de síncope, demostración de compromiso hemodinámico o de falla cardiaca derecha; o bien de laboratorio, a través de la demostración de marcadores serológicos como el proBNP ("Brain Natriuretic Peptide") elevado o un ecocardiograma con evidencias de disfunción ventricular derecha. Ante esta presentación siempre debemos considerar la trombolisis como alternativa terapéutica.

Se ha demostrado la equivalencia de la heparina no fraccionada y las nuevas heparinas de bajo peso molecular en el tratamiento inicial del TEP. Debemos considerar si, las ventajas de esta últimas en relación a la comodidad en su administración. En el caso de un TEP masivo se prefiere el uso de heparinas no fraccionadas. Los nuevos fármacos que inhiben directamente el factor $X$ de la coagulación son alternativas de tratamiento a considerar en algunos grupos de pacientes.

En su presentación sobre la Hipertensión Pulmonar (HP), el Dr. Victor Tapson de la Duke University de Carolina del Norte, se refirió a la clasificación de la OMS 2003 y 2008 que divide a la HP en cinco grupos fundamentales: Grupo I: HP arterial idiopática o asociada a condiciones tales como enfermedades del tejido conectivo, HIV, portopulmonar, cortocircuitos intracardíacos congénitos y drogas entre otras. Grupo II: HP asociada a enfermedad cardiaca izquierda. Grupo III: HP asociada a enfermedad parenquimatosa pulmonar, específicamente EPOC o fibrosis pulmonar. Grupo IV: HP asociada a TEP crónico. Grupo V: HP asociada a otras entidades (misceláneo). La presentación fue principalmente dirigida hacia el grupo I de HP ya que es en este grupo donde se han experimentado los mayores avances diagnóstico-terapéuticos de los últimos años. En términos generales, si bien el mejor examen de pesquisa de esta condición es la ecocardiografía, el cateterismo derecho es fundamental para corroborar el diagnóstico y la caminata de 6 minutos es el mejor examen para el seguimiento. Las tres líneas terapéuticas más relevantes, en las que se basan todas las terapias actualmente aprobadas por la FDA (Food and Drug Administration, USA) son: prostaciclinas (epoprostenol, Iloprost), inhibidores de los receptores de endotelinas (bosentan, ambrisentan, sitaxsentan) y los inhibidores de fosfodiestarasas (sildenafil).

En su exposición sobre hipertensión pulmonar asociada a TEP crónico la Dra. Mónica Zagolín 
hizo énfasis en los principales aspectos de esta entidad, que si bien es infrecuente, se ha demostrado que es más habitual de lo imaginado siendo probablemente subdiagnosticada. En el estudio de Pengo et al ${ }^{3}$, se documenta una incidencia de alrededor de 4\% al cabo de dos años de seguimiento luego de un evento embólico agudo. Para enfatizar lo anterior, cabe destacar que solo un 30\% de los pacientes afectados por esta condición declaran el antecedente de un evento embólico, de modo que muchos de ellos no han presentado un evento agudo en su evolución y esto ha sido lentamente progresivo hasta comprometer la reserva cardiopulmonar y hacerse expresivo, fundamentalmente con disnea, fatigabilidad, palpitaciones, dolor torácico y en las formas más graves, con elementos de falla ventricular derecha y bajo débito, tales como edema y síncopes respectivamente. El diagnóstico es clínico, ecográfico e imagenológico (cintigrafía pulmonar, TAC de tórax y angiografía pulmonar). El cateterismo derecho sella el diagnóstico de hipertensión pulmonar y la alternativa quirúrgica es la endarterectomía pulmonar, procedimiento que puede curar esta condición. En los casos inoperables, existe evidencia en la literatura que la terapia médica con anticoagulación, sildenafil o bosentan puede ser efectiva en el corto y largo plazo.

En su conferencia sobre tratamiento quirúrgico del TEP crónico, el Dr. Ernesto Larraín, enfatizó que la endarterectomía pulmonar es el tratamiento de elección en esta condición, que puede ser curativo, logrando un regreso del paciente a capacidad funcional I, con una reincorporación a sus actividades sociales y laborales. La cirugía corresponde a una endarterectomía pulmonar, procedimiento que se efectúa mediante esternotomía, con paro circulatorio e hipotermia controlada. Los tiempos quirúrgicos de los centros de más experiencia como lo es San Diego en California, son de aproximadamente 15 minutos por lado y la mortalidad operatoria hoy oscila entre 6 y 9\% aunque los centros que se inician en este procedimiento podrían presentar cifras superiores (15 a 20\% de mortalidad postoperatoria). Una de las principales causas de compromiso postoperatorio grave es el edema de reperfusión y la persistencia de la HP. Los pacientes que son mejores candidatos para esta cirugía son aquellos sin comorbilidad relevante, con enfermedad fundamentalmente proximal, central a nivel del tronco o principales ramas de la arteria pulmonar. No se benefician de la cirugía aquellos pacientes con enfermedad distal, es decir, con arteriopatía y remodelación vascular, que es similar a la observada en pacientes con HP tipo I.

Dra. Mónica Zagolín B. * y Dr. Felipe Aller R. *** *Instituto Nacional del Tórax, Santiago de Chile **Coordinador $28^{a}$ Jornadas de Otoño,

Sociedad Chilena de Enfermedades Respiratorias

\section{Bibliografía}

1.- WELLS P S, GINSBERG J S, ANDERSON D R, KEARON C, GENT M, TURPIE A G, et al. Use of a clinical model for safe management of patients with suspected pulmonary embolism. Ann Intern Med 1998; 129: 997-1005.

2.- STEIN P D, WOODARD P K, WEG J G, WAKEFIELD $\mathrm{T}$ W, TAPSON V F, SOSTMAN H D, et al; PIOPED
II investigators. Diagnostic pathways in acute pulmonary embolism: recommendations of the PIOPED II investigators. Am J Med 2006; 119: 1048-55.

3.- PENGO V, LENSING A W, PRINS M H, MARCHIORI A, DAVIDSON B L, TIOZZO F, et al. Thromboembolic Pulmonary Hypertension Study Group. Incidence of chronic thromboembolic pulmonary hypertension after pulmonary embolism. N Engl J Med 2004; 350: 2257-64. 